\title{
Application of the PSAT, an Open Source Software, for Educational and Research Purposes
}

\author{
L. Vanfretti, Member, IEEE, and F. Milano, Member, IEEE
}

\begin{abstract}
This paper describes the authors' and users' experience in the application of a free and open source software for teaching and research in power system analysis and modeling, namely Power System Analysis Toolbox (PSAT). PSAT is currently used in several Universities for undergraduate courses, graduate courses and research. Selected experiences of the application of PSAT for educational and research purposes are discussed in this paper. PSAT has its own web forum, which provides support to students and researchers all around the world. The paper also discusses the contributions of PSAT to power engineering education based on the results of a survey on PSAT filled up by the members of the PSAT web forum.
\end{abstract}

Index Terms-Power system analysis, open-source software, Internet, Matlab, GNU Octave.

\section{INTRODUCTION}

Power system courses deal with complex physical phenomena and detailed mathematical models [1]. More often than not, the complexity of mathematical models deviate students attention from understanding the underlaying main concepts. Furthermore, the computational burden of power system analysis is cumbersome even for small networks, especially if dynamic models are involved. As a result, educational power system software has become a fundamental teaching tool because it helps students to assimilate theoretical issues through graphic visualization of results and the use of userfriendly interfaces.

The deployment of Free and Open Source Software (FOSS) emerges as an option that provides a platform to distribute an educational and research tool obtainable by anyone around the world, and thus, seamlessly creating a community of users/learners that interact and collaborate with each other.

An underlying reason behind the success of a variety of FOSS projects (see for example the Linux, Perl and LATEX experiences) is the freedom given to the users as well as the spirit of collaboration that arises among them. If applied to the power system academic community, the FOSS approach leads to the deployment of tools suitable for education and research, and at the same time to create a community of learners [2].

This paper presents the authors' and users' experience in the usage and development of a specific FOSS, namely Power

Invited Paper, OSS Panel Session, PES GM 2007.

L. Vanfretti is with the Department of Electrical, Computer and Systems Engineering, Rensselaer Polytechnic Institute, Troy, NY, USA. E-mail: vanfrl@rpi.edu

F. Milano is with the Department of Electrical Engineering, University of Castilla-La Mancha, 13071 Ciudad Real, Spain. E-mail: Federico.Milano@uclm.es.
System Analysis Toolbox (PSAT) [3]. PSAT is currently used in several Universities all around the world [4]. Statistics on the users' feedback, which has been collected on the web forum dedicated to PSAT, are also presented in this paper.

As far as the authors know, there are currently only two active FOSS projects for power systems: the PSAT [3] and InterPSS [5]. While PSAT has been designed to provide a tool suitable for research and education, InterPSS is oriented toward consultant activities and power industry applications. Furthermore, InterPSS is currently lacking a comprehensive documentation which makes it not suitable for education. Therefore, PSAT is unique in the sense that is the only tool whose main focus is to support education and research of electric power systems.

PSAT also has its own web forum, which provides support to students and researchers all around the world; this has lead to an almost unique community of learners and a sort of "virtual laboratory" over the Internet.

This paper is organized as follows: Section II presents practical and pedagogical strategies for the development of PSAT. A sample class activity which has been used in teaching an undergraduate course is briefly described in Section III. In Section IV selected documented applications of PSAT as a didactic and research tool are presented, while Section $\mathrm{V}$ describes the PSAT web forum. In particular, subsection V-D presents the results of survey on PSAT proposed to the members of the PSAT forum. Finally, in Section VI, conclusions are duly drawn.

\section{Pedagogical Features of PSAT}

The desired features of pedagogical software and free and open source software are discussed in this section. Afterwards, it is shown how PSAT merges the features of both educational and free and open source software. The pedagogical features implemented in the development of PSAT as an undergraduate teaching tool and as a graduate research-oriented tool are also discussed in this section.

\section{A. Features of pedagogical software}

Software for educational purposes should be user-friendly, easy to use and reliable. In particular, software for power system education should contain an user interface that allows drawing one-line diagrams, displays results and plots time domain simulations.

Most commercial software for power system analysis presents the features described above (see for example PSS/E [6]). However, commercial software has two main drawbacks: it needs a costly license and it is typically very difficult (if 
not impossible) to modify models and/or algorithms provided with the software.

The first drawback basically limits the diffusion of commercial software in developing countries, while the second issue imposes a severe limitation to the software development by Ph.D. students and researchers.

\section{B. Features of FOSS}

Opposite to proprietary software, free and open source software provides the user with the freedom of reading, copying, and modifying the source code. It is also possible with FOSS to redistribute the modified code, with the only condition that the resulting program must also be distributed as free and open source software [7].

Despite initial skepticism shown by commercial software houses, a huge number of FOSS projects have been developed and improved thanks to the cooperation of thousands of users. Some FOSS projects have also obtained worldwide success (see for example the Linux, Perl and $\mathrm{AT}_{\mathrm{E}} \mathrm{X}$ experiences).

If applied to the power system academic community, the FOSS approach would allow deploying tools that are suitable for education and research, and at the same time creating a community of learners [2].

¿From the educational point of view FOSS projects typically have the drawback of being barely understood by an undergraduate student. Even for Ph.D. students to be familiar with the details of large $\mathrm{C}++$ or Java projects would require a lot of time, which should be dedicated to their research topics.

\section{Features of pedagogical FOSS}

An education oriented FOSS should merge the positive features of educational softwares and open source philosophy. Educational free and open source software should have a reasonably user-friendly interface and should be written in a simple and high-level programming language (e.g. Matlab [8] or GNU Octave [9]).

Educational software should develop the learning process and the curiosity of the student. However, only if the code is open can the student explore all the software features. Finally, the students should get used to the idea that knowledge should be free and available to everyone [2].

\section{PSAT, an example of educational FOSS}

PSAT is a Matlab-based FOSS for electric power system simulation and analysis. The command line version of PSAT is also GNU Octave compatible. PSAT includes power flow, continuation power flow, optimal power flow, small signal stability analysis and time domain simulation. All operations can be assessed by means of graphical user interfaces and a SimulinK-based library provides a user friendly tool for network design.

A complete description of PSAT is beyond the main scope of this paper. For interested readers, an in-depth description of all features of PSAT can be found in [3], [4]. A discussion on the usage of PSAT as a Virtual Laboratory is described in [10]. Finally, Appendix A depicts where users can obtain the source code and the documentation of PSAT.

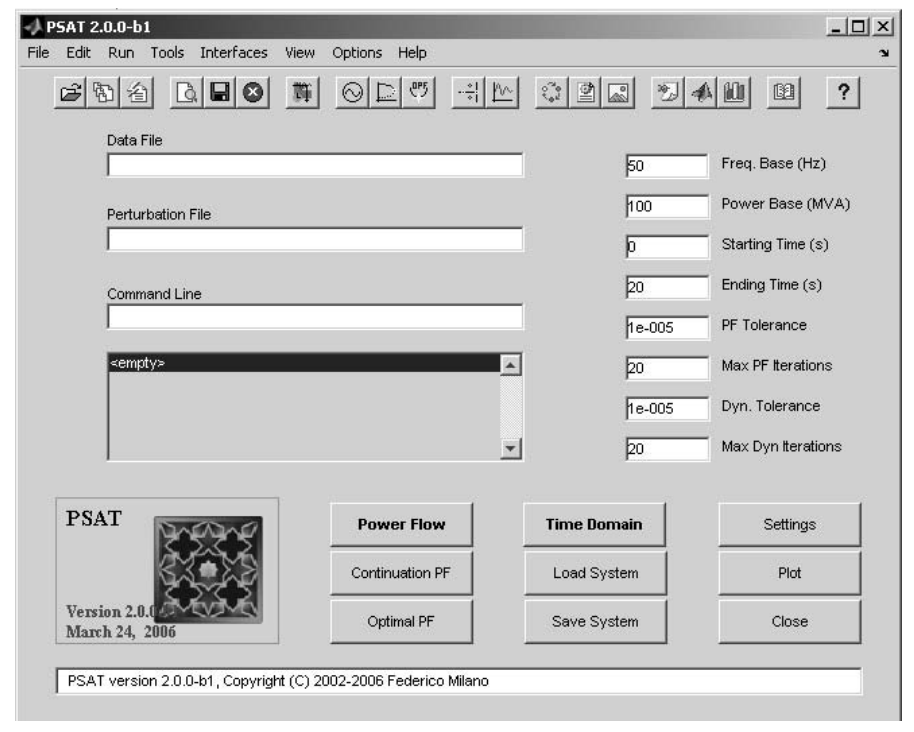

Fig. 1. Main Graphical User Interface of PSAT.

1) Pedagogical and Practical Features: In the authors' experience, PSAT leads to an improvement of the students' learning process in several ways. First, PSAT appears userfriendly to the students because it is based on Matlab, which is typically used by students beginning their first year of undergraduate courses. The students can familiarize themselves with the algorithms not just by using the program but also by reading the code. Furthermore, the educator and the students can modify the algorithms implemented in PSAT and eventually add custom features. Finally, PSAT runs on Linux and GNU Octave; thus there is no need for an expensive license to set up a laboratory using PSAT. This property is particularly relevant in developing countries where the students do not have access to proprietary software.

2) Implementation Considerations: The main practical steps in setting up laboratory experiences or classes based on PSAT can be summarized as follows: system requirements, main goals, and assignment design.

Systems requirements are minimal. The classroom or laboratory should consider an adequate number of personal computers where MATLAB [8] or GNU Octave [9] are properly installed. This consideration is more important in developing nations where the hardware and software should be provided by the universities.

Setting up laboratory classes is also rather simple. PSAT comes with a set of sample test cases that can be used for didactic purposes. If it is necessary to modify or to implement new features, the open source nature of PSAT makes it possible. As it will be described in Section III, power system networks can be extracted from widely used textbooks to design class activities, give assignments, class projects and to teach students how to use toolbox.

The assignments and design activities should be divided into stages of increasing complexity.

1) Students should become familiar with the toolbox. It is important that students get used to the philosophy of the program (e.g. main features, graphical user interface, 


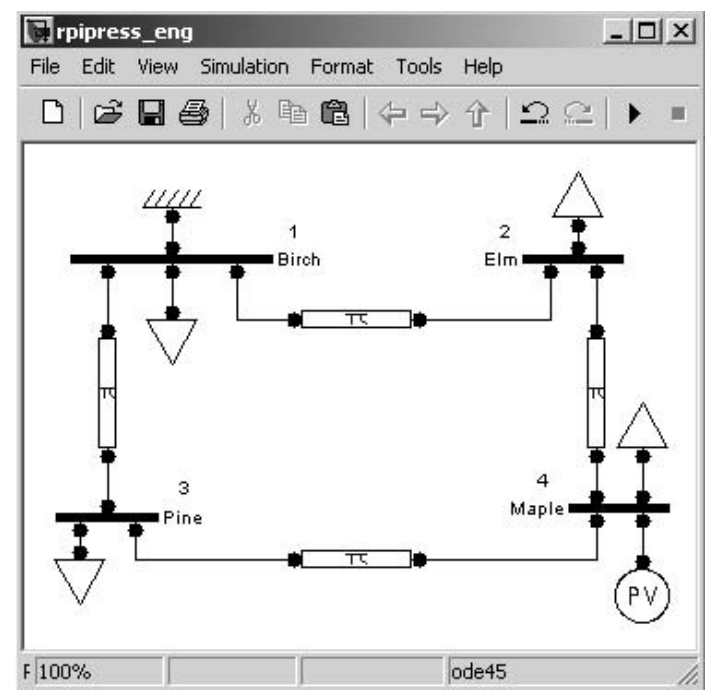

Fig. 2. Small 4-bus Network extracted from [11] and translated into the PSAT-Simulink format.

etc.).

2) The second stage is to familiarize with algorithms and data structures. This will help to design specific class activities, assignments and design activities such as the one illustrated in Section III.

3) For graduate courses, or if the number of student is reduced, it is also possible to ask the students to implement new models and/or plug-ins.

\section{A SAmple Class Activity for Undergraduate TEACHING}

PSAT can be used to exemplify the topics covered in the textbooks recommended for power system analysis courses (e.g. [11], [12]). This can be done by designing computerbased lab activities. In the authors' experience, lab activities stimulate the students' interest in the power system analysis. It is not uncommon that, after attending the lab, students design their own test systems and experiments with the toolbox.

One of the most important topics covered in power system analysis course is power flow analysis. While students generally understand most of the mathematical derivations involved in the development of the Newton-Raphson power flow method, they often show difficulties in understanding the physical meaning and analyzing the results in a "critical" way.

This is likely due to the computational burden involved in the solution of power flow problems which deviates the focus of the students to the computation of each step of the solution method instead of paying attention to the underlaying concepts.

Let us consider the network depicted in Fig. 2, which was extracted from [11] and translated into the PSAT-SIMULINK data format. This network is adequate for a first introduction to power flow analysis. However, the computational burden of a single power flow iteration is high and cannot be done by hand. It is thus necessary to set up a computer-based class and use software to obtain the power flow solution. PSAT can be conveniently adapted for a lab activity that can enhance greatly the students learning process. This simple activity consists of the following steps:

1) Manually obtain the solution for the first iteration of the Newton-Raphson method for the network. Emphasis should be made on the the structure of each of the element of the Jacobian matrix.

2) Construct a PSAT readable data format for the network used in Step 1.

3) Edit PSATs' power flow routine, fm_spf .m, to display the results of each iteration of the Newton-Raphson Method. This can be done by adding the following the commands to the function:

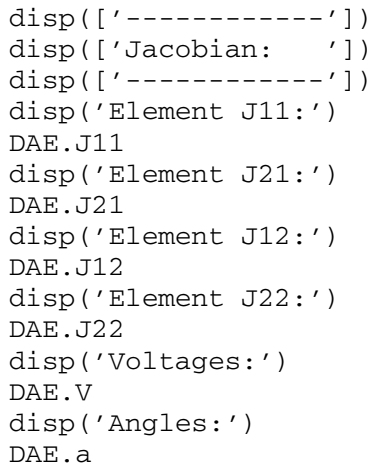

4) Execute the power flow routine of PSAT to obtain a solution to the problem.

5) Compare the first iteration results calculated manually with the one calculated by PSAT, explaining the students how the output structure of the program is arranged so they can observe the changes of the variables for each iteration.

6) Observe the resulting state variables and Jacobian matrix for each iteration. This will offer to the students a clearer idea on how the results are converging to a solution.

7) Compare the solution of the first iteration with the final solution.

8) Vary the tolerance of the power flow convergence and observe how this affects the number of iterations to get the final solution.

9) Vary the load and generator powers and observe how this affects voltages and power flow in transmission lines.

This simple class activity shows how the open source nature of PSAT can help in setting up undergraduate lab activities. PSAT can also be used for graduate courses and research, as is illustrated in the following section.

\section{SAmple EXPERIENCES OF TEACHING AND RESEARCH WITH PSAT}

Figure 3 depicts the diffusion of PSAT all around the world. Observe that, since it is free of charge, the diffusion of PSAT is open to industrialized as well as developing countries.

PSAT is used in several Universities all around the world (see the Appendix J of the PSAT documentation [4] for the recommendation letters). Some examples are: University of Waterloo, Canada; Universidad de San Carlos de Guatemala; Universidad Mariano Galvéz de Guatemala; Universidad Campinas (Unicamp), Brazil; Universidad Centroamericana José 


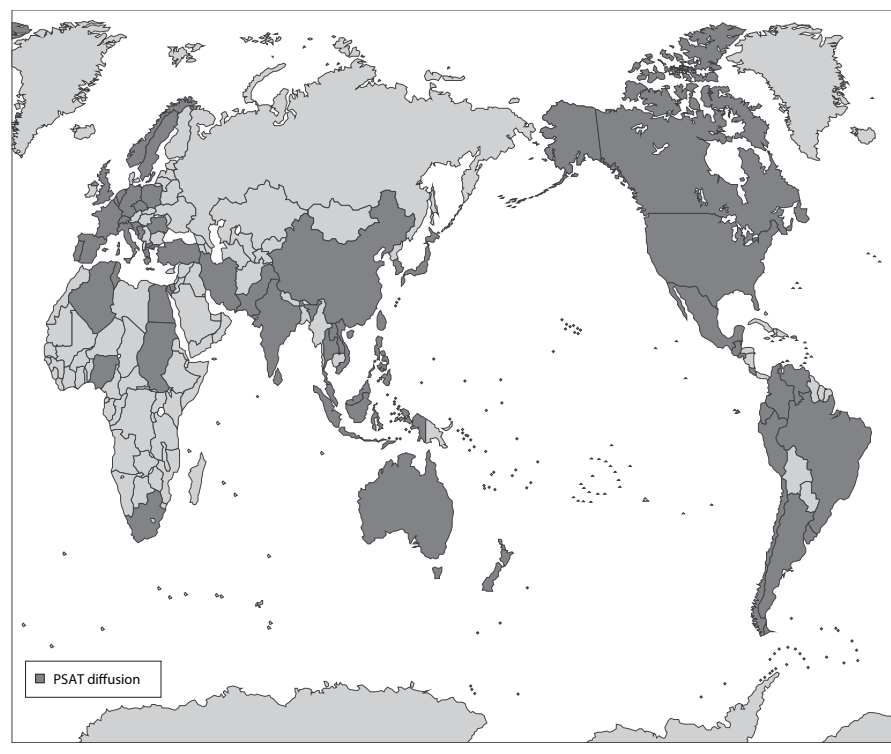

Fig. 3. Documented diffusion of PSAT all around the world.

Simeón Cañas, El Salvador; National Institute of Applied Sciences and Technology, Tunis; University of Maryland, USA; University of New South Wales, Australia; Asian Institute of Technology, Thailand; University of Kocaeli, Turkey; University of Genoa, Italy; Indian Institute of Technology, Kanpur, India; Federal University of Rio de Janeiro; Indian Institute of Technology, Roorkee, India; and Rensselaer Polytechnic Institute, USA.

This section provides the description of three sample experiences of the utilization of PSAT in three Universities for undergraduate teaching, graduate teaching and academic research. The aim of this description is to show the versatility of PSAT and the advantages that free and open source software can offer to the power system academic community. A more comprehensive overview of the applications of PSAT as an educational and research tool is presented in [13].

\section{A. Undergraduate Teaching Experience at Rensselaer Poly- technic Institute (RPI), USA}

PSAT is being used in the undergraduate course EPOW 4010, "Power Engineering Fundamentals" to enhance students learning through computer based assignments. PSAT has been adopted because students can quickly learn how to use it and because of their positive feedback on the program. The sample class activity presented in Section III was used during the Spring semester of 2006. A class project consisting of a design activity was assigned to the students where they had to use PSAT to complete the assignment.

\section{B. Graduate Teaching Experience at the University of Water- loo, Canada}

PSAT is used in the assignment of the graduate course "Power System Computer Applications". The aim of the assignments is to study the IEEE 14-bus test system and find saddle-node, limit-induced and Hopf bifurcations. The simulations required are power flow analysis, continuation power flow analysis, small signal stability analysis and time domain simulations.

\section{Academic Research Experience at the University of Mary- land, USA}

The main feature that has been exploited at this University is the variety of nonlinear power system models that are provided by PSAT. The research topic is detecting and predicting power system instabilities through proper signals obtained by nonlinear analysis. Some results that were obtained using PSAT are reported in [14].

\section{THE PSAT FORUM}

\section{A. History of the Forum}

PSAT has been freely distributed on the Internet since november 2002. The original idea was to share a free and open source Matlab-based software package for power system analysis. Then, a public web forum about PSAT was created on August 2003 on Yahoo! ${ }^{\circledR}$ Groups (more details on the PSAT Forum are given in Appendix A).

\section{B. Activities of the Forum}

The PSAT Forum activities can be categorized as follows: user support, test case repository, and community portal.

1) User support: The main activity of the PSAT Forum is to give user support to all of the program users world wide. User support is given through the reply of messages that are posted on the PSAT Forum. Typically all messages receive a reply. The support provided by the forum is not limited to explaining details of the program or collecting bug reports. Forum moderators clarify doubts, give suggestions, and also help users to solve specific research issues.

2) Test case repository: In the PSAT Forum there is a dedicated area where test power system networks (e.g. IEEE test cases) are provided to the members in the PSAT-Simulink or PSAT data format. Users can also post their own test cases and make them available to other members.

The test system database is an excellent source for students of power system courses at any level because several networks that are proposed in common text books can be found in the Forum [11], [12], [15], [16]. The students can change the configuration of those networks and analyze the effects of the changes. This process clearly enhances the student learning process.

3) Community portal: The PSAT forum also works as a community portal from which the members interact with each other. The spirit of collaboration in the PSAT Forum is quite uncommon in the conservative power system community. The Forum also makes getting contacts that can lead to research or professional collaboration possible.

\section{User Feedback}

With more than 1050 members and more than 3700 posted messages in three years, the web forum dedicated to PSAT shows that a FOSS can become popular among students of 
TABLE I

RELEVANT TOPICS AND THEIR INCIDENCE ON THE PSAT FORUM

\begin{tabular}{l|c}
\hline Topic & Incidence (\%) \\
\hline Slack bus & 16 \\
Infinite bus & 5 \\
Bifurcation theory & 4.5 \\
Continuation Power Flow & 28 \\
Optimal Power Flow & 32 \\
Small signal stability analysis & 10 \\
Time domain simulation & 23.5 \\
N-1 contingency analysis & 2.7 \\
Electricity markets & 5.8 \\
Wind Power & 3.8 \\
SVC & 22 \\
TCSC & 12 \\
STATCOM & 5.6 \\
SSSC & 10.5 \\
UPFC & 14.6 \\
\hline
\end{tabular}

power system courses all around the world. Furthermore, the fact that students subscribe the forum and post messages about their doubts on simulations and power system issues is per se an implicit and clear proof of the usefulness of PSAT and its forum.

Frequent asked questions (FAQs) give an idea of what students find more difficult and thus suggest what can be improved for making the toolbox more user-friendly. From the point of view of the software maintainer, the incidence of FAQs about missing models or algorithms can be used as a valuable database to understand which developments of PSAT could be more useful to the community and what is worth studying. For the sake of illustration, Table I depicts the incidence of some relevant topics in the web forum messages.

\section{PSAT Survey}

This section discusses the results of the PSAT survey that was proposed to the members of the PSAT Forum. The text of the survey is available on the PSAT forum. For the sake of completeness, the survey is depicted in Appendix B.

The job distribution of the members of the PSAT forum is depicted in Fig. II. Note that the majority of the PSAT users are PhD students. Nevertheless, the percentage of researchers, professors, and practitioners is also relevant. Observe also that there are no undergraduate students. This fact was to be expected, since undergraduate students typically use PSAT for an assignment and expect that the teaching assistant (TA) and/or the professor solve their doubts about the program.

Statistics for the survey are depicted in Fig. 4.

Section A of the survey investigates how users found PSAT and the users' impressions of the software and the PSAT Forum. The results show that almost all of the PSAT users found out about the software through the Internet (see poll A.1). This is mainly thanks to the diffusion of PSAT through University web pages (e.g. University of Waterloo, Canada, and University of Castilla - La Mancha, Spain). The responses to poll A. 2 show that up to $40 \%$ of the users decided to adopt
TABLE II

JOBS OF PSAT USERS

\begin{tabular}{l|c}
\hline Job & Percentage (\%) \\
\hline Undergraduate student & 0 \\
Master student or Ph.D. candidate & 53 \\
Professor or researcher & 22 \\
Professional or practitioner & 25 \\
Other & 0 \\
\hline
\end{tabular}

PSAT because it is distributed as a FOSS, while another $40 \%$ of the users decided to use the software because of some of its features. Poll A.3 asks about reasons users subscribed to the PSAT Forum. As was to be expected, about $39 \%$ of the users subscribed to the forum to obtain help on the use of the software. However, the interesting data is that $37 \%$ of the users subscribed to the forum to discuss power systems and about $23 \%$ subscribed to give feedback on the program. This is clear evidence that the PSAT Forum has lead to the creation of a community of learners that share their experience on power systems. Note that this is a unique community in the conservative power engineering field. Finally, note that about $55 \%$ of the users prefer using PSAT among other power system software packages because it is free and open source (see poll A.4).

Section B of the survey aims to understand how PSAT has been used by the users and what impact it had on their work. The replies to polls B. 1 and B. 2 show that about $60 \%$ use PSAT for research, among these about $30 \%$ use standard routines and sample data files provided with PSAT, while $25 \%$ create their own routines and $20 \%$ create their own models. Poll B. 3 addresses the feature of PSAT that has most improved the learning processes of the students. Statistics show that the documentation is the most important source of aid in the learning process with about $35 \%$ of the replies, while the PSAT Forum and user-friendliness of the program are ranked with about $23 \%$ and $20 \%$, respectively. Finally, observe that about $45 \%$ of the users stated that PSAT has been crucial for their research because they did not have access to any other power system software (see poll B.4). This statistic shows one of the most important contributions of PSAT as a research tool.

Finally, polls in Section $\mathrm{C}$ address how users can contribute to PSAT improvement and development. Observe that the user feedback can be really effective due to the open source nature of PSAT. Poll C. 1 shows that users have an extremely positive view of FOSS projects, with almost $98 \%$ of incidence. This result confirms that users find FOSS very useful because they have the freedom to modify the code. Poll C.2 provides useful information on the kind of models and routines that users would like to see implemented in PSAT. Poll C.3 shows that a relevant percentage of users (about 37\%) feel involved in the PSAT development simply because it is an open source project. Poll C.4 shows that the documentation should include a very detailed description of how to modify the program and implement a new Simulink model translation tool. 


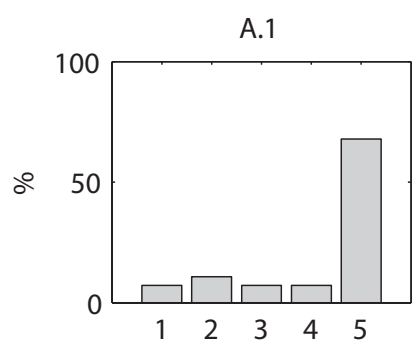

B. 1

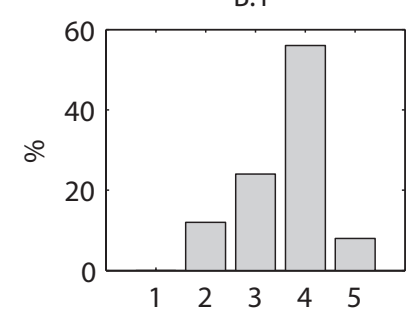

C.1

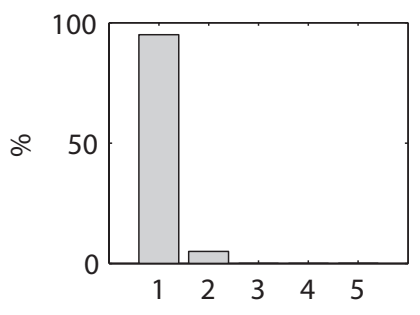

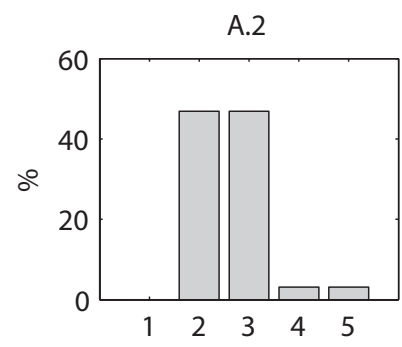
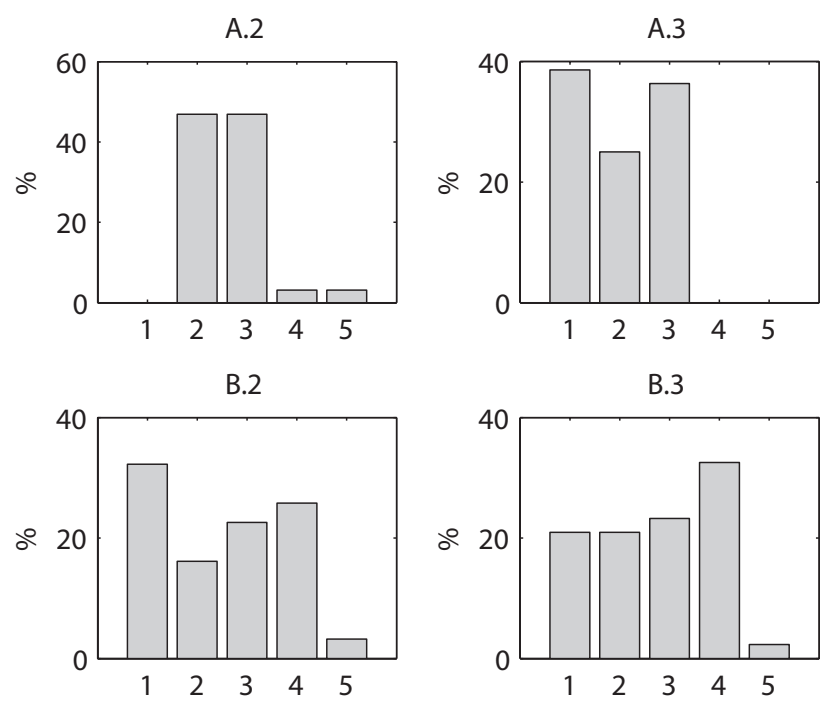

C.2

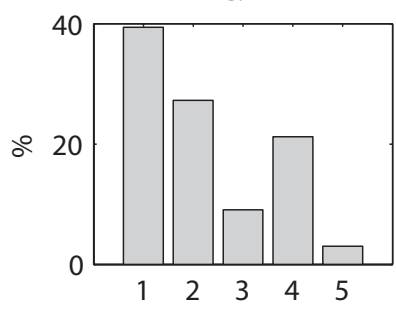

B.3

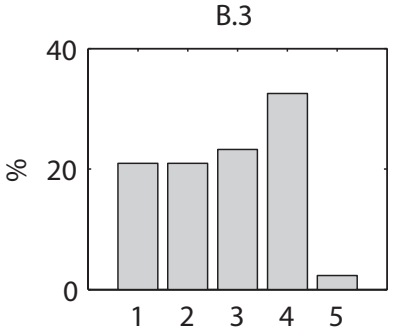

C.3

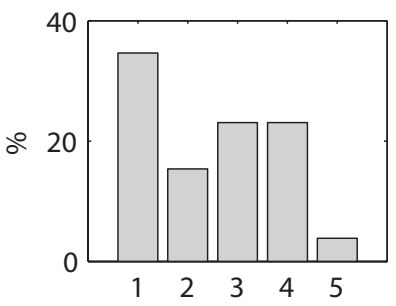

A.4

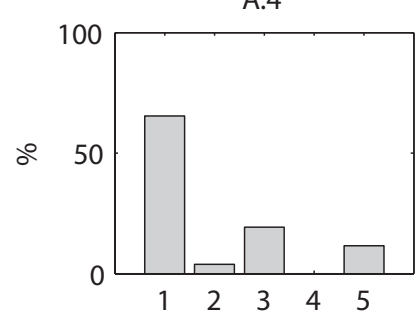

B. 4
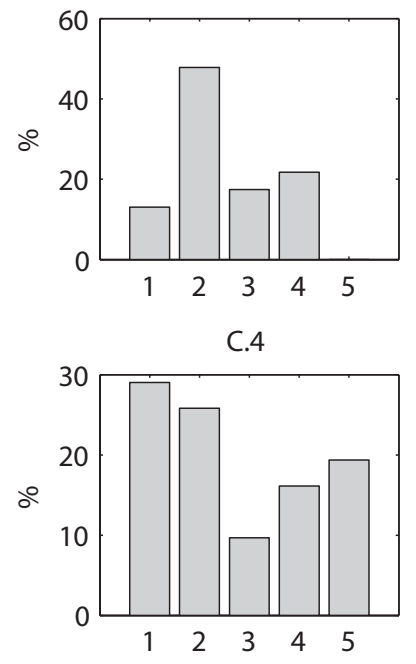

Fig. 4. Statistics for the survey.

\section{CONCLUSIONS}

This paper has presented the authors' experience with PSAT, a free and open source software package for teaching power system analysis, and the research experience of several PSAT users.

Three real-case PSAT-based experiences in teaching and research have been discussed in the paper. The results of a web-based survey on PSAT have been also presented. The survey reveals the great potential of open source software for improving the student learning process as well as stimulating engineering practitioners feedback.

\section{ACKNOWLEDGMENTS}

Federico Milano is partly supported by the Ministry of Science and Education of Spain through CICYT Project DPI2003-01362 and by Junta de Comunidades de Castilla - La Mancha through project PBI-05-053.

\section{APPENDIX A \\ DISTRIBUTION OF PSAT}

This appendix provides information on where to download PSAT. The source code and documentation of PSAT can be downloaded at:

$$
\text { http: //www.power. uwaterloo.ca/ fmilano/ }
$$

This webpage is kindly provided by Prof. C. Cañizares, University of Waterloo, Canada. PSAT is also available at the following mirror at the University of Castilla - La Mancha, Spain:

http: / / www.uclm.es/area/gsee/Web/Federico/

Furthermore, PSAT can be obtained at the PSAT Forum:

http://tech.groups.yahoo.com/group/psatforum/

The websites provide the latest version of the program and its documentation. The Forum also provides test cases, user added libraries, the PSAT survey and latest patches that have not been yet included in the main PSAT distribution.

\section{APPENDIX B PSAT SURVEY}

This appendix depicts the survey that has been proposed to all PSAT users and all members of the PSAT Forum.

\section{PSAT Survey}

0. What is your job?
1) Undergraduate student.
2) Master student or Ph.D. candidate.
3) Professor or researcher.
4) Professional or practitioner in the field of power systems.
5) Other (specify... ).

A. About you and PSAT.
A.1. How did you found out about PSAT?
1) By looking for a power system software package on the Internet.
2) From a friend or a senior student who told me about PSAT.
3) A professor introduced me to PSAT.
4) I read about PSAT on a paper.
5) Other (specify... ). 
A.2. Why did you decide to use PSAT?

1) A professor imposed me to use PSAT.

2) I liked the features of PSAT.

3) Just because PSAT is open source.

4) I had no other available software.

5) Other (specify... ).

A.3. Why did you subscribe to the PSAT Forum?

1) I thought I could get some help.

2) To give a feedback on the program.

3) To discuss about power systems.

4) Actually, I did not subscribe.

5) Other (specify... ).

A.4. Compared to other software packages, what do you think about PSAT?

1) I prefer PSAT because it is open source.

2) I prefer other tools, because PSAT is open source and thus not reliable.

3) PSAT has limited features.

4) I cannot compare because I have used only PSAT.

5) Other (specify... ).

B. How did PSAT help you?

B.1. What have you used PSAT for?

1) For an assignment of an undergraduate course.

2) For an assignment of a graduate course.

3) For my project/thesis.

4) For research

5) Other (specify... ).

B.2. How have you used PSAT?

1) I used standard routines and sample data files.

2) I used standard routines and created my custom data files.

3) I created my custom models.

4) I created my custom routines.

5) Other (specify... ).

B.3. What features of PSAT have helped you to improve your learning process?

1) PSAT is intuitive and easy to use.

2) PSAT has a user-friendly interface.

3) The PSAT forum helped me.

4) The PSAT documentation helped me.

5) Other (specify... ).

B.4. How was the role of PSAT for your course, project or research?

1) Fundamental, because I did not have access to other software.

2) Stimulating, because it is open source.

3) I used PSAT only to compare results obtained with other software.

4) I looked at PSAT only to get ideas.

5) Other (specify... ).

C. How can you help PSAT?

C.1. What is your feeling about open source projects?

1) Great. I can open and modify the source code as I like.

2) It is useful just for people with a high programming skill.

3) Indifferent.

4) Bad. I do not trust "free" software.

5) Other (specify... ).

C.2. What feature of PSAT would you like to be improved, added or changed?

1) A specific algorithm (specify: PF, OPF, etc.).

2) A specific model (specify: load model, AVR, etc.).

3) The GUI (specify: window appearance, Simulink library, etc.)

4) The documentation (specify... ).

5) The web forum (specify... ).

6) Other (specify... . .

C.3. How would you contribute to PSAT?

1) I feel involved in the PSAT development because it is an open source project

2) I would like to contribute but it is too difficult.

3) I would like to, but I do not have time.

4) I am not a programmer, but if I find a bug I will post a message on the Forum.

5) Other (specify... ).

C.4. What would you change in PSAT to feel more involved in its development?
1) The documentation should explain better how to modify and/or change PSAT.

2) If I could use Simulink to create new models, I would contribute.

3) The structure of PSAT should be more modular (e.g. like a Java project).

4) I would like to help, but I need to be guided by the PSAT maintainer.

5) Other (specify... ).

\section{REFERENCES}

[1] M. Kezunovic, G. H. A. Abur, A. Bose, and K. Tomsovic, "The Role of Digital Modeling and Simulation in Power Engineering Education," in IEEE Transactions on Power Systems, no. 1, Feb. 2004, pp. 64-72.

[2] R. M. Stallman, Free Software, Free Society: Selected Essays of Richard M. Stallman. Boston: Free Software Foundation, 2002.

[3] F. Milano, "An Open Source Power System Analysis Toolbox," IEEE Transactions on Power Systems, vol. 20, no. 3, pp. 1199-1206, Aug. 2005.

[4] - "Power System Analysis Toolbox: Documentation for PSAT version 2.0.0," Mar. 2006, available at http://thunderbox.uwaterloo.ca/ fmilano.

[5] M. Zhou, "InterPSS," available at http://www.interpss.org.

[6] Power Technologies Inc., "Online Documentation PSS/E 30," Siemens, Tech. Rep., 2004.

[7] R. M. Stallman, "GNU General Public License," Free Software Foundation, Inc., Tech. Rep., 1991, available at http://www.gnu.org/copyleft/gpl.html.

[8] The MathWorks Inc., Matlab Programming. The MathWorks Inc., 2006, available at http://www.mathworks.com.

[9] J. W. Eaton, GNU Octave Manual. Bristol, UK: Network Theory Ltd., 1997.

[10] F. Milano, L. Vanfretti, and J. C. Morataya, "An Open Source Power System Virtual Laboratory: The PSAT Case and Experience," IEEE Transactions on Education, accepted for pubblication in December 2006.

[11] J. J. Grainger and W. D. Stevenson Jr., Power System Analysis. New York: McGraw-Hill, 1994

[12] J. D. Glover and M. Sarma, Power System Analysis and Design. Boston: PWS Publishing Company, 1994.

[13] F. Milano and L. Vanfretti, "The Experience of PSAT as a FOSS for Power System Education and Research," IEEE Transactions on Education, manuscript submitted in December 2006.

[14] J. Chow, F. F. Wu, and J. Momoh, Applied Mathematics for Restructured Electric Power Systems. Springer-Verlag, 2005, reference in Chapter 8, Instability Monitoring and Control of Power Systems, by E. H. Abed, M. A. Hassouneh and M. S. Saad, from page 171 .

[15] P. W. Sauer and M. A. Pai, Power System Dynamics and Stability. Upper Saddle River, New Jersey: Prentice Hall, 1998.

[16] P. Anderson and A. Fouad, Power System Control and Stability. New York: Wiley-IEEE Press, 2002.

Luigi Vanfretti (M'06) is a $\mathrm{PhD}$ student in the Electrical, Computer, and Systems Engineering Department at Rensselaer Polytechnic Institute, Troy, NY; his supervisor is Professor Joe H. Chow. He received his B.S.E.E. from the San Carlos University of Guatemala in June 2005, from where he also received the Francisco Vela award in February 2006. During fall 2005 he was a Visiting Academic at the Department of Electronics and Electrical Engineering of The University of Glasgow, Scotland. Mr. Vanfretti is a member of IEEE and the IEEE Power Engineering Society. His research interests are dynamics, stability, control, and security of electric power systems.

Federico Milano (M'03) received from the University of Genoa, Italy, the Electrical Engineering degree and the Ph.D. degree in 1999 and 2003, respectively. From 2001 to 2002 he worked at the University of Waterloo, Canada, as a Visiting Scholar. He is currently an assistant Professor at the University of Castilla-La Mancha, Ciudad Real, Spain. His research interests include voltage stability, electricity markets and computer modeling of electric power systems. 